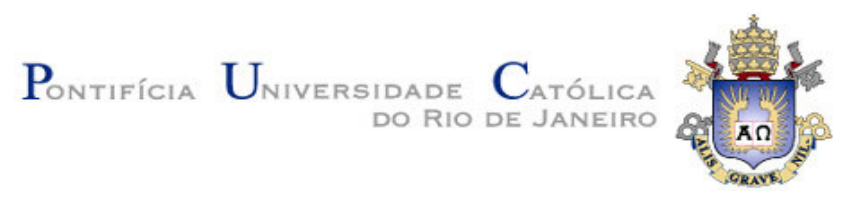

Leonardo Nelo Pedreira

\title{
Proposta para um Sistema de Controle de Armazéns (WCS) com Aplicação em uma Empresa de Pequeno Porte
}

\section{Dissertação de Mestrado}

Dissertação apresentada como requisito parcial para obtenção do título de Mestre em Gerência de Produção pelo Programa de Pós-Graduação em Engenharia Industrial da PUC-Rio.

Orientador: Nélio Domingues Pizzolato - PUC/Rio 


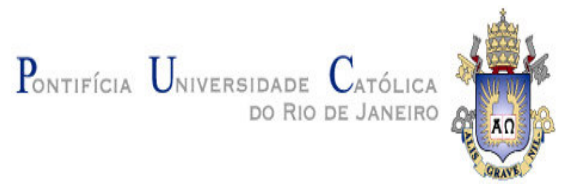

Leonardo Nelo Pedreira

\section{Proposta para um Sistema de Controle de Armazéns (WCS) com Aplicação em uma Empresa de Pequeno}

Porte

\section{Dissertação de Mestrado}

Dissertação apresentada como requisito parcial para obtenção do título de Mestre em Gerência de Produção pelo Programa de Pós-Graduação em Engenharia Industrial da PUC-Rio. Aprovada pela Comissão Examinadora abaixo assinada.

Ph.D. Nélio Domingues Pizzolato

Orientador Departamento de Engenharia Industrial -PUC-Rio

Ph.D. Leonardo Junqueira Lustosa Departamento de Engenharia Industrial -PUC-Rio

D.Sc. Luiz Felipe Roris Rodriguez Scavarda do Carmo

Departamento de Engenharia Industrial -PUC-Rio

Dr-Ing. José Eugênio Leal

Coordenador Setorial do Centro Técnico Científico -PUC-Rio 
Todos os direitos reservados. É proibida a reprodução total ou parcial do trabalho sem autorização da universidade, do autor e do orientador.

Leonardo Nelo Pedreira

Graduou-se em Engenharia de Produção na UEPA (Universidade Estadual do Pará) em 2003.

Ficha Catalográfica

Pedreira, Leonardo Nelo

Proposta para um sistema de controle de armazéns (WCS) com aplicação em uma empresa de pequeno porte / Leonardo Nelo Pedreira ; orientador: Nélio Domingues Pizzolato. - Rio de Janeiro : PUC, Departamento de Engenharia Industrial, 2006.

114 f. ; $30 \mathrm{~cm}$

Dissertação (mestrado) - Pontifícia Universidade Católica do Rio de Janeiro, Departamento de Engenharia Industrial.

Inclui referências bibliográficas.

1. Engenharia industrial - Teses. 2. Sistema de controle de Armazéns (WCS). 3. Logística. 4. Armazenagem. 5. WMS. I. Pizzolato, Nélio Domingues. II. Pontifícia Universidade Católica do Rio de Janeiro. Departamento de Engenharia Industrial. III. Título. 
A Deus, por estar ao meu lado sempre, sendo meu amigo, me dando paz, harmonia, equilíbrio e retidão...... mesmo naqueles momentos em que tudo parecia muito difícil.

Aos meus pais Heron e Virgínia, que jamais mediram esforços para a educação de seus filhos, fonte de amor, carinho e proteção. Obrigado por vocês sempre estenderem a mão para mim. Tenho muito orgulho e amor por ser filho de vocês! É o exemplo de vocês que me dá coragem para lutar!

Ao Erick e Carol, que o exemplo de ajuda, paz e união que nossos pais nos passaram permaneça sempre vivo em nossos corações. lobrigado pelo carinho e torcida que vocês sempre tiveram comigo. Serei eternamente grato!!!

Aos meus avós Sebastião e Eliza "In Memorian” e Rosa e Vírgilio pelo afeto, amor e ensinamento que vocês me passaram desde a época de infância.

Fruto disto é que hoje consigo subir um degrau na escada da vida. 


\section{Agradecimentos}

Ao meu amigo, orientador e professor, Nélio Domingues Pizzolato, por esses dois anos de carinho, dedicação, amizade e ensinamentos.

A CAPES e a Pontifícia Universidade Católica do Rio de Janeiro, em especial ao Departamento de Engenharia Industrial da PUC - Rio, pelo efetivo apoio, pois sem este seria impossível a realização deste trabalho.

O meu sincero agradecimento ao Prof. Leonardo Junqueira Lustosa, não só pela oportunidade de ter realizado o curso de Mestrado nesta casa, como pelos ensinamentos ao longo deste tempo.

Sou muito grato ao Prof. Luiz Felipe Roris Rodriguez Scavarda do Carmo, pela atenção dada e pela competência científica e profissional transmitida, pela amizade, pelo interesse na realização deste trabalho.

O meu sincero agradecimento ao Prof. André Clementino, pela ajuda ao meu ingresso no mestrado e pela confiança depositada. Muito obrigado pela oportunidade.

Aos colegas da Pós-Graduação, pela amizade e feliz convivência neste tempo; que este sentimento de amizade nunca se apague.

Aos Professores e Funcionários do Departamento Industrial da PUC-RIO, pela minha formação na Pós-Graduação.

Aos Professores do Curso de Engenharia de Produção da Universidade Estadual do Pará (UEPA), pela minha formação acadêmica.

A Empresa Montarte pela oportunidade e a equipe de implementação do Sistema WCS, em particular ao amigo e companheiro Bruno Rafael Dias de Lucena.

Sou muito grato à Clarinha e ao Lúcio de Abreu, pelo amor, pela acolhida, torcida ao meu sucesso, carinho e paciência com que me trataram nesses dois anos. Admiro muito a união de vocês!

À Camilla, minha vida, por ter sempre me dado força e amor, em todos os momentos de minha ausência, que pareciam infindáveis. Foi muito difícil estar longe em muitos momentos que para mim eram de grande importância, mas o que mais me encorajou foi à certeza de que fomos feitos um para o outro e que vamos ficar juntos eternamente.

Diversos foram aqueles que contribuíram para a realização dessa pesquisa. Sabendo que corro o risco de esquecer pessoas queridas, gostaria de registrar minha especial gratidão. 


\section{Resumo}

Pedreira, Leonardo Nelo. Proposta para um Sistema de Controle de Armazéns (WCS) com Aplicação em uma Empresa de Pequeno Porte. Rio de Janeiro, 2006. 114p. Dissertação de Mestrado - Departamento de Engenharia Industrial, Pontifícia Universidade Católica do Rio de Janeiro.

A exploração da Logística como atividade estratégica é o resultado da combinação entre a globalização, o aumento da proliferação dos produtos e da utilização de novas tecnologias, como por exemplo, a Tecnologia da Informação. Os Sistemas de Controle de Armazéns (Warehouse Control Systems - WCS) passaram a dar importância à localização do material em um "endereço" arbitrário no armazém, eliminando a necessidade de locais fixos para a armazenagem, permitindo a estocagem onde quer que haja espaço dentro do armazém. Um sistema computacional deve permitir a melhor utilização do espaço. Esta dissertação se propõe a expor a importância da Tecnologia de Informação para a logística, concebendo um projeto em uma empresa de pequeno porte que se enquadre nas técnicas de gestão de armazém, de modo a realizar o mapeamento da atual operação do armazém da empresa. A metodologia empregada foi estudo de caso, utilizando-se o processo de pesquisa-ação, pela necessidade de reavaliação contínua do modelo implementado. Foram realizadas análises das operações antes e durante a implementação do WCS no armazém da referida empresa, além da descrição de suas principais funções e atividades. Foram feitas considerações e discussões práticas sobre a eficiência das operações atuais, bem como de suas funções e objetivos. Foi proposto um novo padrão de execução para as operações de armazenagem com destaque ao endereçamento, separação de pedidos, embalagem, unitização e expedição. Uma base de dados e um novo layout foram constituídos com o objetivo de dar suporte a estas atividades.

\section{Palavras-chave}

Sistema de Controle de Armazéns (WCS), Logística, Armazenagem, WMS. 


\section{Abstract}

Pedreira, Leonardo Nelo. A Proposal for a Warehouse Control System (WCS) with an Application to a Small Size Company. Rio de Janeiro, 2006. 114p. M. Sc. Dissertation - Departamento de Engenharia Industrial, Pontifícia Universidade Católica do Rio de Janeiro.

The exploration of Logistics as a strategic activity is the result of the combination and the use of new technologies, specially the Information Technology. Identified by area, properly registered and controlled by the computer, the Warehouse Control Systems (WCS), defines by an "address" the location of the material in the warehouse, eliminating the traditional existence of fixed places for storage and extending the stockpiling in several sections inside the warehouse,. The main objective of this dissertation is to conceive, in a small company, a project framed in the techniques of storage administration, or in other words, the development of the current operations of the company's warehouse. The methodology used in the elaboration of this work included the action-research process, forced by the need of continuous reevaluation of the implemented model. The main functions and activities of the warehousing operations were described and analyzed before and after the implementation, with the intent to adjust the necessary modifications in the system and its operation. Several considerations and practical discussions are included about the current operation efficiency. A new standard for carrying out warehousing operations, to activities like location issues, separation requests, package, and shipment were suggested. A data base and a new layout have been developed in order to help the realization of these activities.

\section{Keywords}

Warehouse Control Systems (WCS), Logistics, Warehousing, WMS 
Sumário

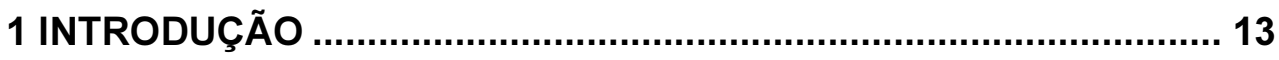

1.1 OBJETIVOS DA PESQUISA …............................................ 14

1.2 DELIMITAÇÃO DO ESCOPO DA DISSERTAÇÃO....................... 16

1.3 METODOLOGIA DA PESQUISA............................................... 17

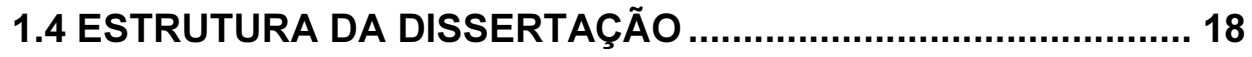

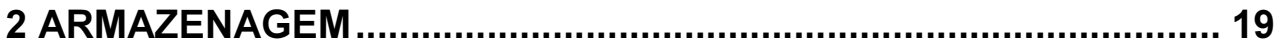

2.1 LOCAIS PARA ARMAZENAGEM ........................................... 22

2.2 A IMPORTÂNCIA DO LAYOUT PARA ARMAZENAGEM......... 23

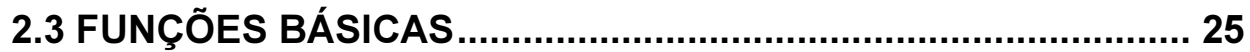

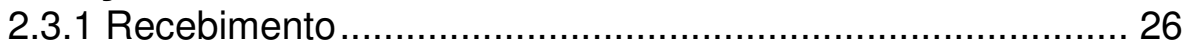

2.3.2 Movimentação.............................................................. 26

2.3.3 Separação de Pedidos ...................................................... 29

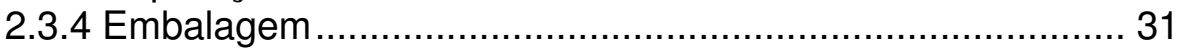

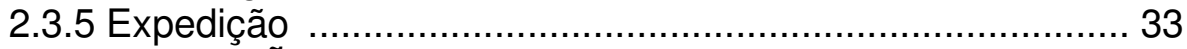

2.4 IDENTIFICAÇÃO DE MATERIAIS....................................... 33

2.5 ENDEREÇAMENTO OU LOCALIZAÇÃO NA ARMAZENAGEM 36

3 TECNOLOGIA DA INFORMAÇÃO 40

3.1 COMO A TECNOLOGIA DA INFORMAÇÃO ESTA AUXILIANDO A LOGÍSTICA 42

3.2 ERP (ENTERPRISE RESOURCE PLANNING OU PLANEJAMENTO DE RECURSOS DE MATERIAIS). 44

3.3 O SISTEMA WCS (WAREHOUSE CONTROL SYSTEM, OU SISTEMA DE CONTROLE DE ARMAZÉNS) ……............................. 45

3.4 O SISTEMA WMS (WAREHOUSE MANAGEMENT SYSTEM, OU SISTEMA DE GERENCIAMENTO DE ARMAZÉNS)............................. 47

3.4.1 Objetivos do Sistema WMS ………................................. 49

3.4.2 Benefícios Ocasionados pelo Sistema WMS ....................... 50

3.4.3 Características e Funcionalidades de um Sistema WMS .... 51

3.5 ARQUITETURA DO BANCO DE DADOS DO SISTEMA

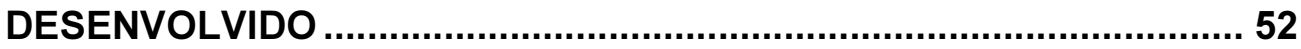

4 ESTUDO DE CASO: EMPRESA MONTARTE ………..................... 54

4.1 CARACTERIZAÇÃO DA EMPRESA..................................... 54

4.2 PRINCIPAIS FUNÇÕES E ATIVIDADES REALIZADAS NO ARMAZÉM 57

4.2.1 Sistema de Informação .................................................... 57

4.2.2 Recebimento de Pedidos no Armazém............................... 58

4.2.3 Recebimento de Mercadorias no Armazém .......................... 59

4.2.4 Movimentação de Materiais no Armazém .............................. 60

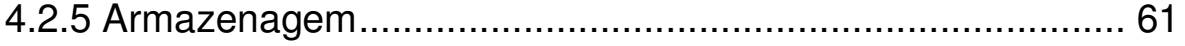




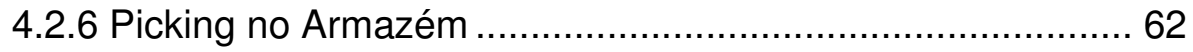

4.2.7 Expedição no Armazém ....................................................... 62

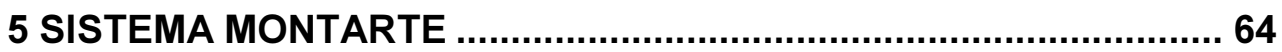

5.1 SISTEMA EXISTENTE NA EMPRESA...................................... 64 5.2 PROCESSO DE DESENVOLVIMENTO DE UM NOVO SISTEMA

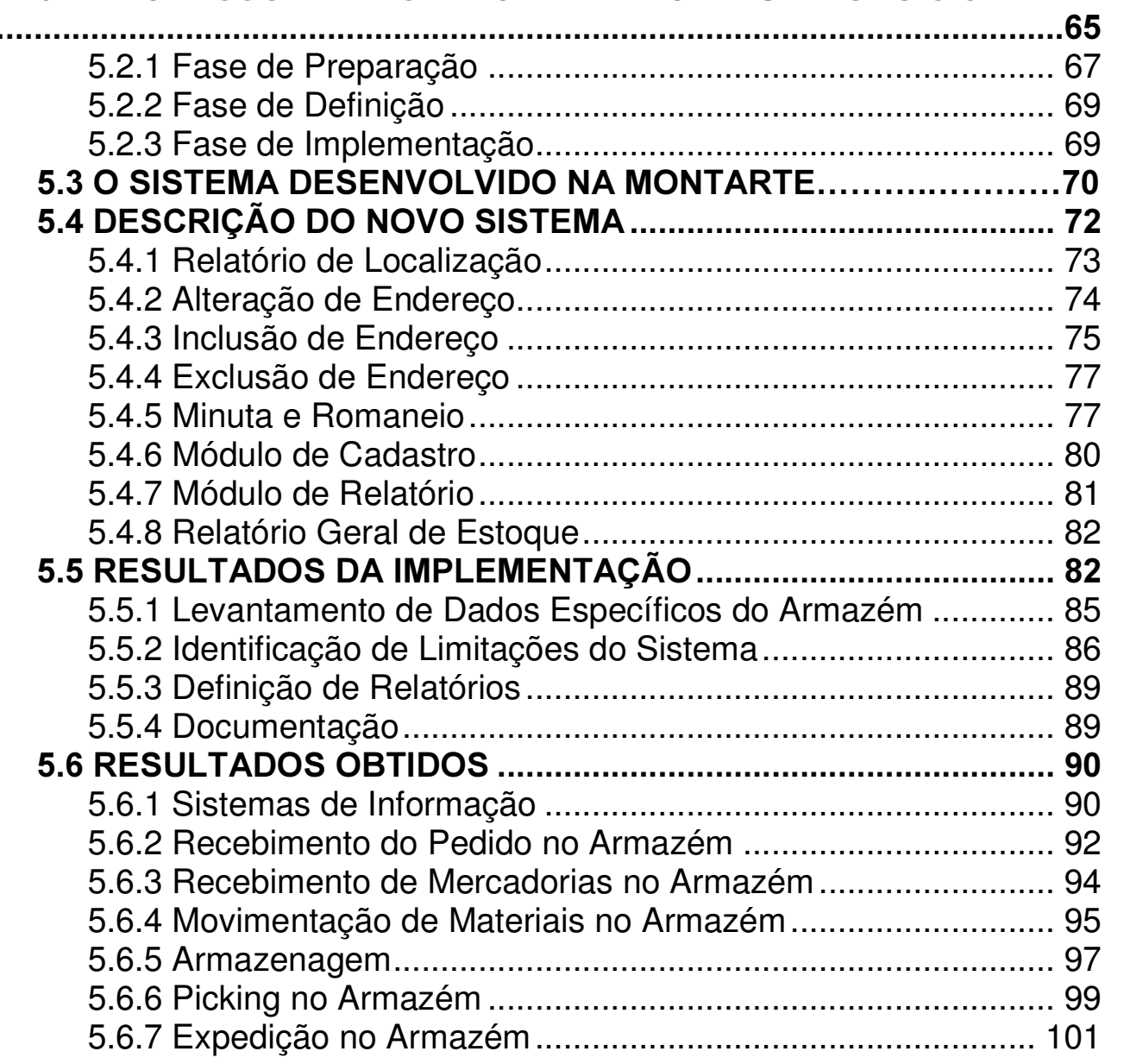

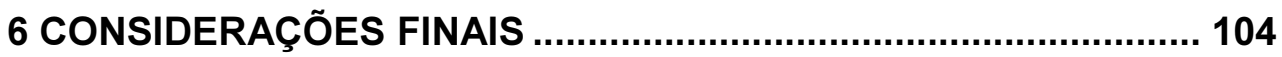

6.1 RECOMENDAÇÕES PARA TRABALHOS FUTUROS.............. 109

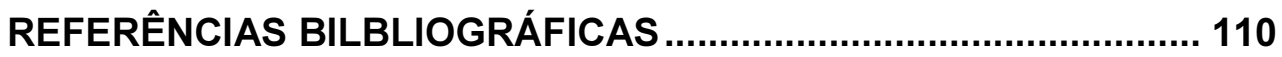

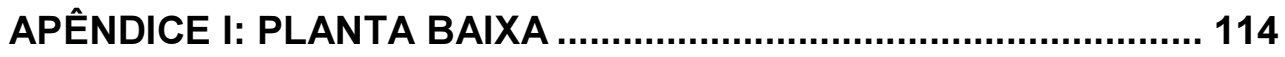




\section{Lista de Figuras}

Figura 1 - Atividade de rastreamento do fluxo do produto através de um

armazém

Figura 2 - Distribuição do tempo do operador.....................................29

Figura 3 - Objetivos (anel externo) e interação da função de

endereçamento no sistema global de distribuição da companhia .....31

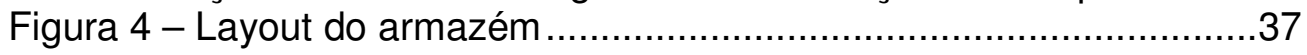

Figura 5 - Esquema de localização para a estrutura porta-palete ............37

Figura 6 - Funcionalidade da informação e suas atividades ......................43

Figura 7 - Arquitetura do banco de dados do sistema montarte ...............53

Figura 8 - Movimentação manual das caixas.......................................60

Figura 9 - Despadronização das caixas nas pilhas ................................60

Figura 10 - Processo de desenvolvimento de projetos de automação ....66

Figura 11 - Sistema de endereçamento de produtos ...............................72

Figura 12 - Relatório de localização...................................................73

Figura 13 - Folha de posicionamento dos produtos disponíveis no

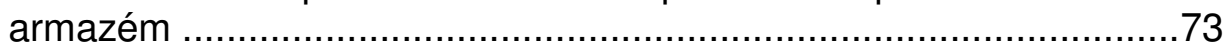

Figura 14 - Tela de alteração de endereço ………………………......75

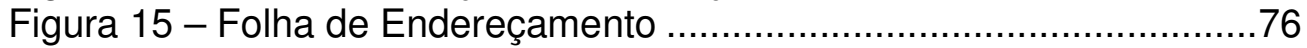

Figura 16 - Tela de inclusão de endereço...........................................76

Figura 17 - Tela de exclusão de endereço...........................................77

Figura 18 - Tela de planejamento e elaboração de minuta........................78

Figura 19 - Tela da elaboração da minuta .............................................79

Figura 20 - Formulário da minuta utilizado na operação...........................79

Figura 21 - Formulário de romaneio utilizado na operação.........................80

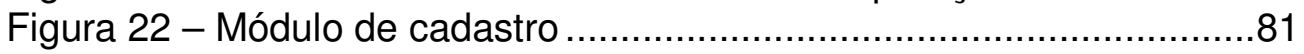

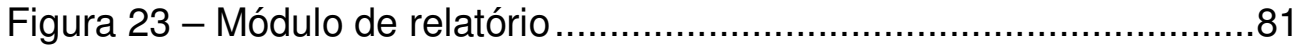

Figura 24 - Folha de relatório geral de estoque ..................................82

Figura 25 - Parâmetros de endereçamento ………………………........ 87

Figura 26 - Produtos de diferente referência armazenados no mesmo setor

Figura 27 - Tabela de controle de pedido

Figura 28 - Relatório gerencial do controle de pedidos...........................92

Figura 29 - Área de embalagem e recebimento.......................................94

Figura 30 - Corredores de um lado ao outro do armazém ........................96

Figura 31 - Carro manual de duas rodas .............................................96

Figura 32 - Carro manual de quatro rodas ..........................................96

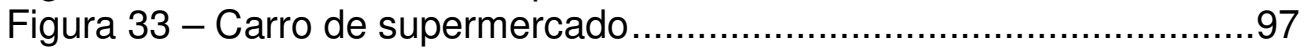

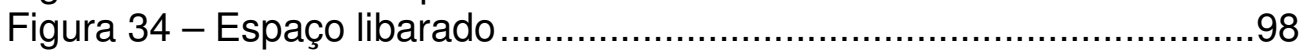

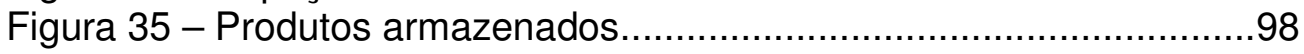

Figura 36 - Layout definido, padronizado e endereçamento de fácil

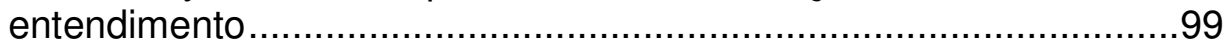

Figura 37 - Formulário de controle de saída ......................................103 


\section{Lista de Tabelas}

TABELA 1 - Principais CaRacterísticas E FunCIONALIDADES DE UM WMS .51

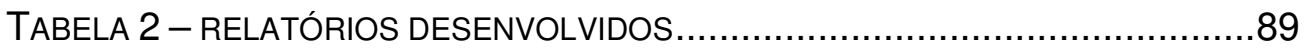

TABELA 3 - PRINCIPAIS IMPACTOS/MODIFICAÇÕES NA OPERAÇÃO ...................108

GRÁFICO 1 - PRODUTIVIDADE DO ARMAZÉM 101 
“..... talvez não haja maior alegria na vida do que encontrarmos meios para vencer nossas fraquezas.

Nós todos conhecemos a embriaguez da vitória e a agonia da derrota.

Encontramos obstáculos e mais obstáculos.

Contudo, com esperança, dignidade, um pouco de loucura e alguma crença em nós mesmos, podemos dar grandes passos na direção da conquista dos nossos objetivos.

O fracasso maior é não tentar.

Muitos, com certeza, desistiram quando com um pouco mais de persistência teriam chegado lá.

Quase sempre, quando tudo parece perdido, quando tudo indica fracasso, nesse momento, Abre-se o caminho....... 